\title{
Editorial
}

Pensar en Movimiento:

Revista de Ciencias del Ejercicio y la Salud EISSN 1659-4436

Vol. 15, № 1, pp. 1- 14

Cierre al 30 de junio de 2017

\section{¿QUÉ ES LA CIENCIA Y HASTA DÓNDE PUEDE LLEGAR?}

\author{
Luis Fernando Aragón-Vargas, Ph.D., FACSM \\ Centro de Investigación en Ciencias del Movimiento Humano \\ Universidad de Costa Rica, Costa Rica \\ Publicado: 28/06/2017
}

¿Tiene la ciencia la última palabra? ¿La puede llegar a tener? En octubre de 2016, la Escuela de Educación Física y Deportes de la Universidad de Costa Rica tuvo como invitado al Dr. René van Woudenberg, filósofo de la ciencia de la Universidad Libre de Amsterdam, quien impartió una clase magistral sobre las presuposiciones de la ciencia. En este número de la revista PENSAR EN MOVIMIENTO se incluyen las versiones en inglés y en español de su manuscrito, luego de pasar por un proceso de revisión por pares. Aprovecho este espacio para compartir con los lectores un tema relacionado que normalmente no se discute entre profesionales del movimiento humano y que, sin embargo, es fundamental para nuestro quehacer científico; además, puede servir como introducción para el manuscrito del Dr. van Woudenberg.

Así pues, presento algunos temas introductorios de filosofía de la ciencia, a saber: la naturaleza de la ciencia, cuáles son sus límites, si estos límites deberían manejarse y cómo. Presento además una lista de buenas prácticas científicas debidamente respaldadas. Como investigador que ha realizado la mayor parte de su trabajo en el rendimiento deportivo y la salud, hago énfasis en las ciencias naturales y, más concretamente, en las ciencias del movimiento humano.

Actualmente abundan las afirmaciones sobre salud y aptitud física, supuestamente fundamentadas en la ciencia. El hecho de que muchas de estas afirmaciones se contradicen entre sí no pareciera molestarle a nadie pues, paradójicamente, más bien es mejor así: cada individuo puede encontrar respaldo científico para sus propios hábitos y creencias. Este elemento es fundamental en una época en que la ciencia goza, en la sociedad occidental, de una posición parecida a la que disfrutaba la religión en la Europa medieval, la cual tenía la última palabra -que uno cuestionaba bajo su propio riesgo. Sin embargo, es necesario preguntarse: ¿es la ciencia realmente confiable, absoluta? ¿Tiene competencia universal como fuente de conocimiento? ¿Debería, más aún, podría ser la guía para la vida? ¿Qué es, exactamente, la ciencia? 
Aunque no pareciera existir una base filosóficamente sólida para esta presuposición, la mayoría de los científicos están de acuerdo en que una teoría o explicación sencilla es mejor que una complicada. Por eso, voy a comenzar con la perspectiva más simple de la ciencia que, según Chalmers (2013 , Capítulo1), es también la más común: vivimos en un mundo natural en el cual las cosas son como son y se comportan de una manera en particular. El método científico es un producto maravilloso que marcó el final de la Edad Media y nos permite a los seres humanos recolectar datos que nos llevan al entendimiento objetivo de ese mundo natural, a la vez que nos posibilita predecir lo que sucederá bajo condiciones específicas. La ciencia ha sido sumamente exitosa por varios siglos; los límites de lo que puede o no contestar parecieran estar dictados únicamente por limitaciones técnicas temporales, como el aumento y la resolución de imágenes de los telescopios y microscopios, la frecuencia de muestreo del video o el almacenamiento y procesamiento de datos. Todo lo que vale la pena conocerse debería estudiarse científicamente; la ciencia debería tener la palabra final en todo. Una vez más, esta es una visión de la ciencia bastante generalizada en Occidente.

Ciertamente la ciencia ha sido muy exitosa, pero resulta que las cosas son ligeramente más complicadas de lo que presenté en el párrafo anterior. Al menos eso es lo que los filósofos de la ciencia nos plantean a los científicos. Sus argumentos son bastante sólidos: en primer lugar, no existe una definición clara que les permita a los humanos intelectualmente honestos distinguir entre ciencia y no ciencia, aún después de varios intentos importantes (cf. Moreland, 1989, Capítulo 1). En segundo lugar, el método científico—una serie de pasos claramente definidos que llevan desde las preguntas iniciales hasta el desarrollo de una teoría-no existe. En tercer lugar, no es posible de manera alguna para la ciencia el afirmar que ha llegado a la verdad: no hay forma de probar nada de manera absoluta. Por último, pareciera que la ciencia ni siquiera puede justificar su propia credibilidad sin tomar prestados elementos de otras disciplinas como la filosofía. En las páginas siguientes, intentaré discutir algunos de estos problemas y brindar recomendaciones para algunas buenas prácticas científicas.

¿Es acaso tentativo todo el conocimiento? ¿Qué tal el conocimiento no científico? ¿Existe la verdad objetiva o existen formas múltiples de entender el mundo natural, todas igualmente válidas siempre y cuando nos hagan felices y permitan que las cosas sigan funcionando? En el proceso de responder a estas preguntas debo partir de mis propias suposiciones: 1) creo que no es posible tener una discusión racional sin admitir primero que todos partimos de nuestras presuposiciones-la ciencia definitivamente lo hace (cf. Moreland, 1989); 2) creo que existe un mundo natural, físico, que efectivamente se comporta de manera predecible y que los seres humanos tenemos la capacidad de percibirlo y comprenderlo; 3) junto a muchos científicos del siglo XVII y muchos de mis contemporáneos, creo que el mundo natural fue creado por un ser racional, personal, que lo dotó de muchas de sus propias características como orden, racionalidad, consistencia, belleza y verdad; 4) creo, además, que la ciencia es una disciplina muy amplia que nos puede revelar muchísimo acerca de nuestro mundo natural, pero no es la única que nos puede dar conocimiento importante y confiable, además de que no puede hacerlo aislada de otras disciplinas. Más aún, creo que las respuestas sinceras a las preguntas más importantes de la vida están fuera de los límites de la ciencia (Lennox, 2009; Medawar, 1984; Van Woudenberg, 2016).

Partiendo del supuesto de que la ciencia no puede estudiar el mundo natural aislada de otras -2 - 
disciplinas, mi perspectiva es que debe ser iluminada por la filosofía. En una cita de John Kekes (Kekes, 1980, Nature of philosophy, pp. 156-157), Moreland presenta una lista de presuposiciones importantes de la ciencia:

La ciencia está comprometida con varias presuposiciones: que la naturaleza existe, tiene un orden descubrible y es uniforme, son presuposiciones existenciales de la ciencia; las distinciones entre tiempo y espacio, causa y efecto, el observador y lo observado, lo real y lo aparente, el orden y el caos, son presuposiciones de clasificación; mientras tanto la comprobación intersubjetiva, la cuantificabilidad y la posibilidad de tener acceso público a los datos son presuposiciones metodológicas. Algunas presuposiciones axiológicas son el reporte honesto de los resultados, el valor de capturar los hechos correctamente y la escrupulosidad para evitar el error experimental 0 de observación. Si abandonáramos cualquiera de estas presuposiciones sería imposible ejercer la ciencia como la conocemos. Y sin embargo la aceptación de las presuposiciones no puede darse por descontada, pues cada una ha sido cuestionada y hay alternativas a nuestra disposición. (Moreland, $\underline{1989}$, p. 109. Traducción libre)

Una de las características principales de la ciencia pareciera ser que, al ser ejercida o practicada por los seres humanos, no es posible ser totalmente imparciales y objetivos, por más chocante que esto pueda parecerle al científico ingenuo. Para los positivistas y los empiricistas, sería ideal si fuéramos capaces de recolectar datos libres de las restricciones de cualquier teoría, pero varios autores han presentado argumentos muy sólidos de que esto es imposible (Chalmers, 2013; Ratzsch, 2000). Es absolutamente imprescindible partir de algunos supuestos y del conocimiento existente para poder ejecutar una recolección de datos que sea productiva: las teorías a menudo están implícitas en la forma en que medimos los fenómenos, en la escogencia de instrumentos de medición o hasta en la selección que hacemos de los fenómenos que se van a medir. En pocas palabras, no es posible que el científico ejerza su profesión independientemente de sus propios intereses y perspectivas. De este modo, su ciencia se verá impactada no solamente por las teorías más directamente relacionadas con su campo de trabajo, sino inclusive por su cosmovisión.

Según lo que plantea Ratzsch (2000), este ingrediente humano de la ciencia fue rescatado en cierta forma por Thomas Kuhn: más que un elemento no deseable pero inevitable, la aplicación de los valores humanos se puede ver como fundamental para la práctica de la ciencia. La posición de Ratzsch es que "la filosofía de la ciencia contemporánea ha estado buscando un punto medio en el cual la razón, la observación y la objetividad ocupan su debido lugar, pero en el cual el factor humano es al menos eso—un factor" (Ratzsch, 2000, Capítulo 3, último párrafo. Traducción libre).

Ahora bien, ¿cuál es la labor del científico? ¿Nos corresponde buscar la verdad o simplemente intentar encontrar una explicación lo suficientemente buena? Platón fue cuidadoso al referirse a este tema, al afirmar en su Timaeus: "si podemos elaborar descripciones no menos probables que cualquier otra, deberíamos darnos por satisfechos, teniendo presente que tanto yo, quien habla, como ustedes, los jueces, somos solamente humanos (...) No nos corresponde buscar más allá de esto." (McGrew, Alspector-Kelly y Allhoff, 2009, p. 27. Traducción libre). Poco después, aparentemente, la 
balanza se había inclinado rápidamente hacia la certeza, como se puede ver en el planteamiento de Aristóteles en sus Analíticos Posteriores. Pero el equilibrio cambió nuevamente más adelante: "Un avance filosófico crítico que acompañó a la revolución científica fue el cambio del concepto aristotélico de ciencia como conocimiento absolutamente cierto derivado de los principios fundamentales, a un concepto más moderado de la ciencia como disciplina racional pero falible" (McGrew et al., 2009, p. 9. Traducción libre).

Por otra parte, Moisés Maimónides expresó una perspectiva curiosamente práctica de la verdad y la ciencia en su obra La Guía de Perplejos, al afirmar que el propósito del astrónomo "no es informarnos cómo son verdaderamente las esferas, sino plantear un sistema astronómico en el cual sería posible que los movimientos fueran circulares y uniformes y que correspondieran con lo que se puede percibir por la vista, independientemente de si las cosas son así en realidad." (McGrew et al., $\underline{2009}$, p. 84. Traducción libre). En otras palabras, no importa si el modelo no es verdadero, siempre y cuando calce con los datos observados. Esta pareciera ser la perspectiva de muchos científicos contemporáneos, pues ejercen un instrumentalismo práctico aunque no necesariamente se adhieran formalmente a esa filosofía.

Yo estoy en desacuerdo con dicha posición: ciertamente la ciencia no tiene la capacidad de aportar suficientes pruebas de que hemos alcanzado la verdad-"la ciencia experimental aporta la prueba no del logista, sino del abogado.--" (Decaen, 2012, p. 26), pero yo sí estoy convencido de que la verdad existe y que deberíamos utilizar nuestras mentes para intentar alcanzarla, aun si nuestros esfuerzos son como la asíntota de Medawar: "pues no puede existir la certeza apodíctica en la ciencia, no hay certeza finalmente absoluta fuera del alcance de la crítica." (Medawar, 1984, p. 5. Traducción libre). En nuestra búsqueda de la verdad, quisiéramos creer que la ciencia avanza y que dicho avance significa que nos acercamos cada vez más a ella.

El significado y relevancia de la verdad es solamente una de muchas fuentes de desacuerdo entre los filósofos. A pesar de ellas, he logrado identificar varios puntos importantes comunes a la mayoría de las filosofías de la ciencia. Uno de ellos es la imposibilidad de saber cuándo se ha alcanzado la verdad, la cual se mencionó en el párrafo anterior. También existe la convicción de que debería haber un constante esfuerzo por garantizar la objetividad, la racionalidad y el empiricismo. Hay una creencia generalizada y necesaria en la uniformidad de la naturaleza (Van Woudenberg, 2017). E independientemente de la perspectiva personal, ninguna filosofía de la ciencia puede explicar todos y cada uno de los acontecimientos que conforman la rica historia de los descubrimientos científicos.

Para concluir esta sección debo añadir que el solo hecho de que exista tantísimo debate entre las distintas filosofías de la ciencia refleja que esta última está lejos de haber alcanzado la perfección y que no tiene la capacidad de orientarnos en la dirección correcta por sí sola. La ciencia es limitada, a pesar de que algunos científicos no se comporten como si lo fuera.

\section{Los límites de la ciencia}

Aun el estudio de los límites de la ciencia es considerablemente más complicado de lo que podríamos imaginar. Existen otros límites además de los que quisiera presentar más a profundidad, a saber: si realmente existen áreas del conocimiento en las cuales la ciencia simplemente no puede ofrecer una respuesta (Sir Peter Medawar, 1984, las llama "las cosas primeras y últimas"). Algunos de 
ellos tienen que ver con la autolimitación del crecimiento, con la capacidad tecnológica o con la insuficiencia cognitiva; en esta línea, Medawar declara que "no existe un límite a la capacidad de la ciencia de contestar el tipo de preguntas que la ciencia puede contestar" (Medawar, 1984, p. 86. Traducción libre). La cita suena desafortunadamente circular, pero el contexto muestra que el autor quiso decir que todas las preguntas dentro del dominio de la ciencia pueden contestarse y serán contestadas tarde o temprano.

Ahora bien, algunos límites prácticos se deben a la disponibilidad de recursos económicos, sociales o ambientales, a pesar de que algunos de estos podrían ajustarse por medio de políticas. Otros límites prácticos y externos tienen que ver con experimentos o procedimientos en los cuales la ciencia no debería embarcarse por razones éticas, un campo muy amplio y controversial en el cual, como en otros, la línea no está claramente demarcada. En el resto de este apartado intentaré centrarme únicamente en lo que la ciencia, por su naturaleza, puede y no puede decirnos acerca del mundo en que vivimos. Mi lista, por supuesto, no será exhaustiva en cuanto a lo primero (sería una tarea imposible), ni tampoco en cuanto a lo último.

Quizás el límite más importante de la ciencia es que no puede justificar su propia credibilidad, como se mencionó anteriormente, ni tampoco puede justificar por qué deberían descartarse otras disciplinas legítimas y racionales como la filosofía o la teología. La declaración de que solamente la ciencia puede llevar a la verdad no se puede deducir de la ciencia misma; se trata de una declaración que se refuta a sí misma (Lennox, 2009). Lo mismo sucede con la afirmación que dice "solamente lo que se puede conocer científicamente o cuantificado y puesto a prueba empíricamente es verdadero y racional" (Moreland, 1989, p. 107). El punto es que existen muchos casos en los cuales la ciencia no es la fuente de nuestro conocimiento, pero eso no significa que la razón esté ausente y que la evidencia deje de ser relevante (Lennox, 2009). La ciencia no tiene el monopolio del conocimiento racional.

La ciencia, por su misma naturaleza, no abarca todos los tipos de conocimiento racional. Van Woudenberg (2016) presenta algunos ejemplos de lo que él llama el conocimiento irreduciblemente extracientífico. Se trata de hechos o realidades que yo conozco, pero cuyo conocimiento adquirí sin ayuda alguna de la ciencia; de hecho, la ciencia ni siquiera me podría permitir conocerlos. Se trata de mi entendimiento de que mentir es malo, o de que tengo la obligación de cuidar a mis hijos y mis padres ancianos. La condición de fundamento para ese conocimiento no puede ser satisfecha por nada que sea científico. Se deben analizar aquellas preguntas que están fuera de la ciencia, como por ejemplo ¿qué es la virtud moral? o ¿cómo adquirimos conocimiento moral? Es importante estudiarlas porque, como escribió Moreland (1989):

(...) nuestra cultura está inundada de tal forma por el cientificismo-en breve, la perspectiva de que solamente aquello que la ciencia nos dice que es verdadero o racional es, efectivamente, verdadero o racional-y ha existido un énfasis tan pragmático en la ciencia en el sistema educativo (...) que existe un mito cultural ampliamente difundido según el cual toda pregunta como las mencionadas anteriormente es un asunto de opinión privada (p. 46. Traducción libre).

En este sentido, un límite para la ciencia sería "una explicación o respuesta a algún problema, -5 - 
que reside fuera de las fronteras de la explicación científica y es de naturaleza cognitiva, esto es, se trata en principio de un asunto racional cuya solución puede ser cierta o aproximadamente cierta" (Moreland, $\underline{1989}$, p. 105. Traducción libre). O si no, en las palabras de van Woudenberg, la ciencia se ve limitada por el conocimiento irreduciblemente extracientífico, aquellas creencias verdaderas que están bien fundamentadas pero cuya condición de fundamento no puede provenir de la ciencia.

No es posible poner demasiado énfasis en este límite: existen preguntas que están fuera del dominio de la ciencia. Uno siempre debería preguntarse: ¿cuál es la justificación científica, o al menos racional, para creer que la ciencia será capaz de explicarlo todo? Pareciera que no existe ninguna. En sus argumentos a favor de la ciencia y en contra de la religión o la teología, algunos científicos contemporáneos recurren con frecuencia, de manera explícita o implícita, al concepto de un "dios de las lagunas". Este concepto fue introducido por Lucrecio en el primer siglo d.C. y ha llegado a ser muy popular entre los científicos agnósticos y ateos: conforme los seres humanos somos capaces de explicar más y más fenómenos naturales que anteriormente se le atribuían a "los dioses", esos dioses se van quedando sin trabajo (McGrew et al., 2009, p. 14).

Otra manera de plantearlo es que el concepto de "dios" es útil para explicar lo inexplicable, pero conforme la ciencia vaya logrando explicar cada vez más cosas, llegará el momento en que la explicación "dios lo hizo" ya no será necesaria. A menudo se da una extrapolación automática, no justificada, de este argumento al mundo no observable, a lo sobrenatural. Se comete un grave error en este tipo de razonamiento al no reconocer que, como la ciencia decide limitarse a los conceptos naturalistas, o a aquello puramente mecanicista o materialista, es indispensable aceptar que la mayoría de las preguntas sobre valores, moralidad, religión, filosofía y otras áreas, no serán de su competencia. Según Ratzsch (2000), la mayoría de los filósofos de la ciencia aceptan estos límites, pero algunos de ellos no lo hacen, y en muchos casos la motivación tiene un sabor antirreligioso. La afirmación generalizada de que el Dios del cristianismo es un "dios de las lagunas" es ciertamente un paso fuera de las fronteras de la ciencia, a pesar de que desafortunadamente exista un fundamento histórico para hacerla.

Por lo tanto, la ciencia tiene un alcance limitado debido a que existe conocimiento racional que no es científico. Otra perspectiva sobre este punto es conforme al tipo de preguntas que deben contestarse: la ciencia plantea una amplia gama de preguntas e intenta contestarlas, pero como se mencionó anteriormente de manera breve, sencillamente no le es posible contestar las preguntas últimas, tales como ¿por qué existimos?, ¿cómo fue que todo empezó?, ¿son acaso libres los seres humanos? Algunos han propuesto que estas preguntas son irrelevantes, pero si eso es cierto, ¿por qué surgen una y otra vez? Como declara van Woudenberg, "dado que las preguntas últimas continúan pareciéndonos significativas e importantes, y que la ciencia no parece tener cómo abordarlas, parece que todo lo que se deduce es que sería incorrecto e irrazonable recurrir a la ciencia en busca de las 'respuestas últimas'” (2016, p. 14).

Ahora bien, no solamente no es posible para la ciencia contestar las preguntas últimas, sino que tampoco le es posible contestar preguntas sobre hechos crudos. Esto se refiere a explicaciones finales o últimas fundamentadas en las leyes universales, las cuales no explican por qué se aplican dichas leyes en vez de otras. Un ejemplo de esta categoría de hechos crudos es la constante gravitacional: sabemos cómo aplicarla y nos resulta sumamente útil cuando calculamos el movimiento humano o los viajes espaciales, pero la ciencia no puede explicar qué es la gravedad. Los hechos 
crudos constituyen un límite para las ciencias naturales (van Woudenberg, 2016).

Existe otra amplia categoría de límites de la ciencia que he insinuado ya dos veces en este editorial, pero que ahora quisiera describir con un poco más de detalles: la existencia de presuposiciones de la ciencia. La ciencia no puede existir, no se puede ejercer, a menos que se parta de ciertas presuposiciones básicas a las cuales no se puede llegar científicamente. Esto se ha discutido entre filósofos de la ciencia con gran profundidad; por eso invité al Dr. René van Woudenberg a compartir con nuestra revista un manuscrito sobre el tema, el cual se encuentra en este número tanto en inglés como en español. En ese manuscrito se puede encontrar una presentación más profunda y detallada del tema.

Quizás el mejor ejemplo de las presuposiciones de la ciencia es el principio de uniformidad, al cual David Hume llamó originalmente "el problema de la inducción": en vista de que los procedimientos $A$ y $B$, bajo las circunstancias $C$ y $D$, han producido el resultado $S$ un número considerable de veces, seguirá siendo así. Este principio, como ya mencioné en mi editorial anterior, no puede ser probado por medio alguno que esté a disposición de los seres humanos. Las enormes debilidades de esta presuposición fueron captadas con claridad por Dell Ratzsch:

Así que la uniformidad no puede predecir, no se puede someter a prueba, no posee riesgo empírico, puede tergiversarse para amoldarse casi a cualquier cosa, recibe una protección preferencial y descansa en última instancia sobre consideraciones filosóficas. Y sin embargo el principio de uniformidad no solamente es legítimamente científico, sino que es absolutamente esencial para la ciencia (Ratzsch, 2000, Methodological Naturalism, párrafo 10. Traducción libre).

Aparentemente, se tiende a juzgar más rigurosamente a otras disciplinas... Otra presuposición clave consiste en que podemos confiar en nuestras habilidades cognitivas como la percepción, la razón y la memoria, pero para poner a prueba su confiabilidad primero tenemos que confiar en ellas. William Alston le llamó a esto "circularidad epistémica" (van Woudenberg, 2016, p. 11). Entonces, resulta que no es posible respaldar científicamente la confiabilidad de aquellas habilidades cognitivas esenciales para el quehacer científico.

La última categoría de límites a la cual quisiera referirme tiene que ver con las normas para la elección de teoría. Existe una gran variedad de normas que nos ayudan a escoger entre dos o más teorías que compiten entre sí, pero no hay un acuerdo sobre su importancia relativa; más aún, no existe consenso sobre cuáles deberían incluirse y cuáles no. La ciencia misma no puede indicarnos cómo escoger entre las teorías sin recurrir a la filosofía y toda suerte de convicciones y creencias extracientíficas. En la siguiente sección sobre la mejor ciencia se presentan algunos ejemplos de las normas para la elección de teoría, un tema especialmente importante para los editores de revistas científicas, pues incide en las decisiones de rechazar o aceptar manuscritos para su publicación.

Después de plantear que los límites sí existen, quisiera proponer brevemente cómo deberían manejarse. Existen al menos dos frentes. Para comenzar, los científicos debemos reflexionar acerca de nuestra disciplina y hacer un esfuerzo sincero por respetar sus fronteras, o, si no, esforzarnos por complementarla con herramientas, principios y expertos de otras disciplinas. Luego, en la medida en 
que distintas personas irrespeten los límites, ya sea por tener perspectivas radicalmente distintas sobre la ciencia o por la tendencia natural que tenemos de llevar descuidadamente nuestras afirmaciones y convicciones tan lejos como se nos permita, la comunidad científica deberá asumir su responsabilidad de arbitraje, de la misma forma en que los editores y revisores arbitran la calidad de las publicaciones en las revistas científicas. Existen muchos ejemplos de científicos y filósofos que, sin llegar al extremo de la censura algunas veces presente en las revistas científicas, están cumpliendo un papel en el manejo de los límites de la ciencia al mantener estos temas sobre la mesa y contribuir a la discusión con sus argumentos bien fundamentados. Todo científico responsable debe estar al tanto de las discusiones en torno a los límites de la ciencia.

\section{¿Existe una mejor ciencia?}

Las tres características deseables de la ciencia o de sus teorías, según Ratzsch (2000), son la objetividad, la racionalidad y el empiricismo. A pesar de que ya se habían mencionado, no fueron definidas, en parte porque algunos filósofos de la ciencia de mucho renombre le han dedicado páginas innumerables a la clarificación de estos términos. Para este editorial, y consciente del riesgo de una excesiva simplificación, se entenderá por racionalidad la expectativa de un orden, de que el mundo es regular o uniforme y que exhibe patrones que son comprensibles para el científico; empiricismo o empirismo tiene que ver con hechos que son observables, esto es, con la posibilidad de verificar los hechos mediante la medición o la experimentación; finalmente, objetividad significa que la interpretación de esos hechos o mediciones no debería variar sustancialmente de un científico a otro.

Ahora bien, estas características no pueden darse por sentadas, pues existe todo tipo de amenazas a cada una de ellas. Sin embargo, hay muchas maneras de fomentarlas. En ese sentido, debería ser posible distinguir la ciencia débil de la ciencia más sólida. A fin de cuentas, los científicos tienen la necesidad de saber cómo hacer mejor su ciencia, los editores de las revistas deben ser capaces de rechazar los artículos científicos menos sólidos, las agencias patrocinadoras de investigación deberían fundamentar sus decisiones en la calidad científica de las propuestas recibidas además de sus propias políticas, y aún el público con suficiente educación tiene la necesidad de poder reconocer la ciencia más firme.

Por lo tanto, mientras es imposible elaborar una lista de verificación con los requisitos suficientes y necesarios, algunas pautas deberían ayudar a distinguir la ciencia más fuerte de la más débil. En este editorial se presentan dichas pautas en forma de tabla (ver Tablas 1 y 2), clasificadas según se relacionan con la calidad de una teoría o con la calidad de los experimentos específicos o sus elementos de evidencia. Para utilizar la terminología de Stephen Wykstra citada por van Woudenberg (2016), la Tabla 1 presenta las pautas teóricas, mientras la Tabla 2 presenta las metodológicas. Dentro de cada categoría, he intentado presentarlas en orden ascendente de sofisticación, de manera que las cualidades más sofisticadas tienden a no ser tan ampliamente aceptadas. En las tablas también intenté identificar si cada cualidad reforzaría naturalmente la objetividad, la racionalidad o el empiricismo. 
Tabla 1

Pautas para distinguir la calidad de la ciencia según los aspectos teóricos

\begin{tabular}{|c|c|c|}
\hline Cualidad o característica & Ciencia más sólida & Ciencia más débil \\
\hline $\begin{array}{l}01 \text { Sencillez } \\
\text { Racionalidad } \\
\text { (la consideramos } \\
\text { sencilla porque puede } \\
\text { ser comprendida por la } \\
\text { mente humana) }\end{array}$ & $\begin{array}{l}\text { Típicamente es preferible una teoría más } \\
\text { sencilla, que explica todos los fenómenos } \\
\text { observados, a una teoría compleja. }\end{array}$ & $\begin{array}{l}\text { Una teoría que se vuelve } \\
\text { complicada para lograr } \\
\text { acomodar algunas discrepancias } \\
\text { en la evidencia. }\end{array}$ \\
\hline $\begin{array}{l}02 \text { Asociación y } \\
\text { sucesión } \\
\text { Racionalidad }\end{array}$ & $\begin{array}{l}\text { Cuando dos o más fenómenos ocurren } \\
\text { juntos (conjunción o contigüidad). Se } \\
\text { considera un primer paso básico, pero } \\
\text { nunca es suficiente para establecer } \\
\text { causalidad. El caso se vuelve un poco } \\
\text { más sólido si los fenómenos ocurren } \\
\text { siempre en el mismo orden (sucesión). }\end{array}$ & $\begin{array}{l}\text { La asociación solamente se ha } \\
\text { observado una vez o unas pocas } \\
\text { veces. }\end{array}$ \\
\hline $\begin{array}{l}03 \text { Estar libre de } \\
\text { imposibilidades } \\
\text { Racionalidad }\end{array}$ & Por sí sola, no es una cualidad fuerte. & $\begin{array}{l}\text { Cuando la descripción de algún } \\
\text { fenómeno está libre de } \\
\text { imposibilidades (propuesta por } \\
\text { Aristóteles en Meteorología, } \\
\text { según la presenta McMullin, } \\
\underline{2013} \text {, p. 153). }\end{array}$ \\
\hline $\begin{array}{l}\text { 04 Coherencia o } \\
\text { consistencia } \\
\text { Racionalidad y } \\
\text { empiricismo }\end{array}$ & $\begin{array}{l}\text { Existen muchas observaciones } \\
\text { disponibles y todas ellas (las apparentia } \\
\text { de Buridan) están en armonía con la } \\
\text { explicación. } \\
\text { Se considera significativamente más } \\
\text { fuerte si se han realizado varios intentos } \\
\text { infructuosos de falsificar la teoría o } \\
\text { explicación. } \\
\text { Chalmers (2013) le llama a esto "el } \\
\text { proceso de poner a prueba las } \\
\text { afirmaciones frente a la evidencia". }\end{array}$ & $\begin{array}{l}\text { Solamente hay unas pocas } \\
\text { observaciones disponibles, a } \\
\text { pesar de ser consistentes con la } \\
\text { explicación. } \\
\text { TAMBIÉN cuando una o más de } \\
\text { las observaciones disponibles no } \\
\text { son consistentes con la explica- } \\
\text { ción, pero se les ajusta ad hoc } \\
\text { para alcanzar la consistencia. } \\
\text { En vez de poner a prueba las } \\
\text { afirmaciones frente a la } \\
\text { evidencia, aquellas se acomodan } \\
\text { para calzar con esta. }\end{array}$ \\
\hline
\end{tabular}




\begin{tabular}{ll}
\hline Cualidad o característica & Ciencia más sólida \\
\hline 05 Falsabilidad & $\begin{array}{l}\text { Relacionada con la anterior. La teoría o } \\
\text { hipótesis debe tener un alto riesgo de ser }\end{array}$ \\
Empiricismo y & $\begin{array}{l}\text { falseada o refutada. Reconoce la } \\
\text { falibilidad de la ciencia. }\end{array}$
\end{tabular}

\section{Ciencia más débil}

Una teoría sin contenido informativo, la cual, por lo tanto, no puede ser refutada. Según Karl Popper, el psicoanálisis, por ejemplo, nunca podrá equivocarse, pues es suficientemente flexible para acomodarse a cualquier hecho. Al no poder eliminar o refutar nada, no es capaz de explicar realmente nada.

\begin{tabular}{llll}
\hline 06 Capacidad de & $\begin{array}{l}\text { La teoría es capaz de predecir resultados } \\
\text { predicción }\end{array}$ & $\begin{array}{l}\text { Las predicciones } \\
\text { nuevos, desconocidos o inclusive } \\
\text { inesperados (McMullin, } \underline{2013}, \text { p. 180, }\end{array}$ & $\begin{array}{l}\text { sonservadoras, con muy pocas } \\
\text { probabilidades de fallar. }\end{array}$ \\
Racionalidad y & Medawar, $\underline{1985}$, p. 4). \\
empiricismo & $\begin{array}{l}\text { Las predicciones o explicaciones tienen } \\
\text { un alto grado de exactitud. }\end{array}$
\end{tabular}

\begin{tabular}{lll}
\hline 07 Explicaciones & Existen otras explicaciones alternativas, & La única fortaleza de la teoría \\
alternativas & pero la que se ha propuesto muestra propuesta es la insuficiencia de \\
& evidencia suficientemente buena y es todas las explicaciones \\
Empiricismo, & coherente. & alternativas.
\end{tabular}
racionalidad

\begin{tabular}{l}
\hline $08 \quad$ Campo de $\quad \begin{array}{l}\text { Un ámbito más amplio es preferible a un } \\
\text { ámbito extremadamente limitado. }\end{array}$ \\
aplicación
\end{tabular}

Objetividad y racionalidad

Existe la posibilidad de dos
extremos problemáticos. En primer lugar, un ámbito extremadamente limitado; pero en segundo lugar, un campo de aplicación demasiado amplio: la aplicación se extiende más allá de los límites fundamentados (extrapolación no fundamentada) o, como lo plantea la partición de teorías de Mayo, las teorías generalizan a un grado mayor de lo que se justificaría conforme a la evidencia disponible.

09 Fecundidad en el
proceso de guiar
nuevas
investigaciones

Empiricismo

\begin{abstract}
Se considera más sólida una teoría con respecto a su rival si apunta con mayor claridad a nuevas áreas de investigación, si fomenta el progreso.
\end{abstract}

La teoría de un experimento en particular es estéril, lleva a un callejón sin salida. 


\begin{tabular}{|c|c|c|}
\hline Cualidad o característica & Ciencia más sólida & Ciencia más débil \\
\hline $\begin{array}{l}10 \text { Verificación de las } \\
\text { reglas de } \\
\text { correspondencia } \\
\text { Racionalidad }\end{array}$ & $\begin{array}{l}\text { Aunque la confirmación de las reglas de } \\
\text { correspondencia no es posible, sí es } \\
\text { posible lograr una muy buena } \\
\text { aproximación, cuando hay disponibilidad } \\
\text { de reglas de correspondencia } \\
\text { inconfundibles conectadas con los } \\
\text { mismos asuntos teóricos, o cuando las } \\
\text { teorías existentes en otras áreas } \\
\text { totalmente diferentes se pueden enlazar } \\
\text { teóricamente (Ratzsch, 2000). } \\
\text { Existe convergencia entre varias pruebas } \\
\text { independientes: cuando distintos } \\
\text { fenómenos que se pueden probar } \\
\text { independientemente se pueden explicar y } \\
\text { predecir mediante la misma teoría } \\
\text { (Artigas, } 2001, \text { p. 200). }\end{array}$ & $\begin{array}{l}\text { Cuando ni siquiera es posible } \\
\text { intentar la confirmación de las } \\
\text { reglas de correspondencia. }\end{array}$ \\
\hline $\begin{array}{l}11 \text { Consistencia con el } \\
\text { conocimiento } \\
\text { extracientífico } \\
\text { Racionalidad }\end{array}$ & $\begin{array}{l}\text { Las teorías son consistentes con el } \\
\text { conocimiento extracientífico generalmente } \\
\text { aceptado. } \\
\text { Las teorías son compatibles con las } \\
\text { creencias metafísicas bien } \\
\text { fundamentadas (Ratzsch } 2000 \text {, cuando se } \\
\text { refiere a la Racionalidad de la Ciencia de } \\
\text { Newton-Smith, 1981). }\end{array}$ & $\begin{array}{l}\text { Las teorías están en conflicto } \\
\text { con el } \quad \text { conocimiento } \\
\text { extracientífico } \\
\text { aceptado. }\end{array}$ \\
\hline
\end{tabular}

Es necesario señalar que estos no son factores empíricos conforme los definen los convencionalistas y los pragmatistas. Se encuentran más bien en la línea de algunos de los valores de la ciencia de Kuhn: exactitud empírica, consistencia, amplitud de alcance, sencillez y fecundidad (Ratzsch, 2000, Rationality, párr. 8), o en la línea de lo generalmente aceptado como deseable para las teorías: suficiencia empírica, sencillez, buen ajuste con otras teorías, que genere descubrimientos inesperados, fecundidad, encaje observacional, historial, fluidez, consistencia interna y compatibilidad con creencias metafísicas bien fundamentadas (Ratzsch, 2000, Confirmation, párr. 17). Asimismo, están relacionados con los cinco criterios de Artigas para evaluar teorías en la investigación científica normal: poder explicativo, poder predictivo, exactitud de las explicaciones y predicciones, convergencia de pruebas variadas e independientes, y respaldo mutuo. En el contexto de la Teoría de la Racionalidad Científica, van Woudenberg (2016) las llama "normas para la elección de teoría".

Como se explicó en el último límite de la ciencia presentado en la sección anterior, el consenso no es claro acerca de estas normas, y cuando se obliga a los científicos a escoger entre dos teorías aparentemente equivalentes, ellos no tienen criterios científicos absolutos o definitivos para hacer su elección. Le asignan diferente peso o importancia a cada una de las cualidades que se presentan en estas tablas y llegan hasta el extremo de descartar algunas de ellas. 
Tabla 2

Pautas para distinguir la calidad de la ciencia según los aspectos metodológicos

\begin{tabular}{ll}
\hline Cualidad o característica & Ciencia más sólida \\
\hline 01 El fenómeno bajo estudio & La afirmación se puede someter al \\
es observable & escrutinio empírico, es "observable" \\
(cuantificable, medible) y & (cuantificable) mediante diferentes \\
verificable & métodos y por diferentes personas.
\end{tabular}

Empiricismo y objetividad

\section{Ciencia más débil}

La afirmación solamente es observable por un instrumento en particular y la funcionalidad de este instrumento se fundamenta en una teoría relacionada con el fenómeno bajo estudio.
02 Espectro y variedad de la Un amplio espectro de fenómenos, evidencia. Coincidencia (Chalmers, 2013)

Empiricismo, racionalidad y (Chalmers, 2013, capítulo 17). según los describen varias pruebas genuinas cualitativamente distintas, coinciden para respaldar la teoría
Las pruebas que respaldan la teoría son muy limitadas en cuanto al tipo o la cantidad. objetividad

\begin{tabular}{ll}
\hline 03 Buen proceso inductivo & No solamente se cumplen los Se limita a cumplir los \\
& procedimientos inductivos apropiados, procedimientos \\
Racionalidad y empiricismo & sino que además se intenta excluir apropiados. \\
& otros factores causales relevantes y \\
& se somete a prueba la invariabilidad \\
& de cualquier pretensión de \\
& correlación, como lo recomendó \\
& Francis Bacon.
\end{tabular}

04 Reproducibilidad La reproducción o repetición de un La reproducibilidad rigurosa experimento por otros investigadores podría no ser posible. Aún si lo Objetividad y empiricismo es altamente deseable y fuera, las agencias generalmente se considera una patrocinadoras a menudo característica necesaria de la ciencia. rechazan los experimentos que no añaden nuevas perspectivas. Podría ser necesario justificar cómo los resultados de nuevos experimentos son una reproducción de resultados previos.

\begin{tabular}{lll}
\hline 05 Calidad del experimento & $\begin{array}{l}\text { Resultados de experimentos } \\
\text { ejecutados apropiadamente pero que } \\
\text { además son relevantes y significativos } \\
\text { Racionalidad }\end{array}$ & $\begin{array}{l}\text { Resultados de experimentos } \\
\text { ejecutados apropiadamente pero } \\
\text { (cf. Chalmers, 2013). }\end{array}$ \\
& $\begin{array}{l}\text { pues no añaden nada al estado } \\
\text { actual del conocimiento. }\end{array}$
\end{tabular}




\begin{tabular}{|c|c|c|}
\hline Cualidad o característica & Ciencia más sólida & Ciencia más débil \\
\hline $\begin{array}{l}06 \text { Manejo de resultados } \\
\text { únicos o atípicos } \\
\text { Objetividad y empiricismo }\end{array}$ & $\begin{array}{l}\text { En un estudio que reporta resultados } \\
\text { atípicos es necesario tener más } \\
\text { cuidado: el diseño experimental debe } \\
\text { ser sólido y un grupo independiente } \\
\text { debe reproducir posteriormente los } \\
\text { resultados. } \\
\text { Se ha sometido a cualquier elemento } \\
\text { auxiliar necesario a pruebas } \\
\text { independientes de la teoría que se } \\
\text { está presentando (Chalmers, } \underline{2013} \text {, } \\
\text { cap. 17). } \\
\text { Los autores aportan referencias o } \\
\text { ejemplos que respaldan o ponen a } \\
\text { prueba los elementos auxiliares de } \\
\text { manera independiente. }\end{array}$ & $\begin{array}{l}\text { Se introducen elementos } \\
\text { auxiliares, pero no se les somete } \\
\text { a prueba de manera } \\
\text { independiente. Más bien, se } \\
\text { limitan a acomodar la teoría a la } \\
\text { evidencia. }\end{array}$ \\
\hline $\begin{array}{l}07 \text { Conformidad con los } \\
\text { criterios aceptados en el } \\
\text { contexto de disciplinas } \\
\text { específicas } \\
\text { Racionalidad, objetividad }\end{array}$ & $\begin{array}{l}\text { Además de todas las cualidades } \\
\text { enumeradas anteriormente, que se } \\
\text { aplican a todas las ciencias naturales, } \\
\text { existen aquellos criterios específicos, } \\
\text { actualmente aceptados, para cada } \\
\text { disciplina. Los métodos utilizados en } \\
\text { la investigación cumplen con los } \\
\text { criterios específicos preferidos por la } \\
\text { comunidad científica en ese campo en } \\
\text { particular. Por ejemplo, en ciencias } \\
\text { del ejercicio, los estudios con seres } \\
\text { humanos son "de doble ciego". }\end{array}$ & $\begin{array}{l}\text { Cuando los métodos no cumplen } \\
\text { con los criterios comúnmente } \\
\text { aceptados en la disciplina y } \\
\text { además el experimento o teoría } \\
\text { no es sólido en otros aspectos. }\end{array}$ \\
\hline
\end{tabular}

En resumen, en este editorial he presentado brevemente tres ideas principales relevantes al quehacer científico. En primer lugar, expliqué que el concepto popular de ciencia es a menudo demasiado simplista, casi infantil, pues no toma en cuenta las profundas discusiones entre los filósofos que se han tomado en serio este tema. Concretamente, propuse que no existe una definición clara que les permita a los humanos honestos y pensantes distinguir entre ciencia y no ciencia; que no hay consenso sobre el método científico, como una serie de pasos claramente definidos que llevan desde las preguntas iniciales hasta el desarrollo de una teoría; que la ciencia no puede afirmar que ha llegado a la verdad (aunque creo que la verdad sí existe), pues nada se puede probar de manera absoluta; y que el quehacer científico es imposible sin partir de presuposiciones no científicas. En segundo lugar, he planteado que la ciencia tiene límites que los científicos debemos conocer y respetar, si queremos ser intelectualmente honestos. Por último, aunque no exista un consenso entre los científicos para establecer la frontera entre ciencia y no ciencia, planteé que sí existen criterios o cualidades de carácter teórico y metodológico que nos permiten distinguir entre la ciencia más sólida y la más débil. 
Se agradece de manera especial a Peter Distelzweig, Ph.D., profesor del Departamento de Filosofía de la Universidad de St. Thomas, St. Paul, Minnesota, por su paciencia y apoyo con este manuscrito. La investigación para este editorial fue posible gracias a una licencia sabática de la Universidad de Costa Rica en el primer semestre de 2015.

\section{REFERENCIAS}

Artigas, M. (2001). The Mind of the Universe: Understanding Science and Religion. Recuperado de https://www.amazon.com/Mind-Universe-Understanding-Science-Religion/dp/1890151548

Chalmers, A. F. (2013). What is this thing called Science? ( $4^{\text {ta }}$ ed.). Queensland, Australia: University of Queensland Press. [Versión Kindle para PC]. Recuperado de https://www.amazon.com/WhatThis-Thing-Called-Science/dp/162466038X

Decaen, C. A. (2012). Galileo, Exegete of Scripture vs. Rome, Advocate of Science. Second Spring International Journal of Faith Culture, (15), 16-27. Recuperado de https://www.secondspring.co.uk/product/issue-fifteen-faith-and-science-2/

Lennox, J. C. (2009). God's Undertaker - Has Science Buried God? (2 ${ }^{\text {da }}$ ed.). Recuperado de https://www.amazon.co.uk/d/Books/Gods-Undertaker-Has-Science-Buried-JohnLennox/0745953719

McGrew, T., Alspector-Kelly, M., \& Allhoff, F. (2009). Philosophy of Science: an Historical Anthology. Recuperado https://books.google.co.cr/books/about/Philosophy of Science.html?id=srfaAAAAMAAJ\&redir e $\underline{\mathrm{SC}=\mathrm{Y}}$

McMullin, E. (2013). The inference that makes science. Zygon Journal of Religion \& Science, 48(1), 143-191. Recuperado de http://onlinelibrary.wiley.com/doi/10.1111/j.14679744.2012.01319.x/abstract

Medawar, P. (1984). The Limits of Science. Recuperado de https://books.google.co.cr/books/about/The Limits of Science.html?id=r5ppAAAAMAAJ\&redir e $\underline{\mathrm{SC}=\mathrm{Y}}$

Moreland, J. P. (1989). Christianity and the Nature of Science - A Philosophical Investigation. Recuperado de https://books.google.co.cr/books/about/Christianity and the nature of science.html?id=QjoNA QAAMAAJ\&redir esc=y

Ratzsch, D. (2000). Science \& its Limits: The Natural Sciences in Christian Perspective (2 ${ }^{\text {da }}$ ed.). Downers Grove, IL: InterVarsity Press. [Versión Kindle para PC]. Recuperado de https://www.amazon.com/Science-Its-Limits-Perspective-Philosophy-ebook/dp/B001Q3LA9K/

Van Woudenberg, R. (2016). Los límites de la Ciencia y su relación con la fe cristiana. Clase Magistral del Académico Plantinga, Universidad de Notre Dame, 2008. Traducción al español recuperada de http://kerwa.ucr.ac.cr/handle/10669/29393.

Van Woudenberg, R. (2017). Las presuposiciones de la ciencia (del movimiento humano). Pensar en Movimiento: Revista de Ciencias del Ejercicio y la Salud, 15(1):17-33. Recuperado de http://dx.doi.org/10.15517/pensarmov.v15i1.29046 